\title{
Numerical study of the laminar premixed flame stabilization on a slot burner: comparison between detailed and FGM models
}

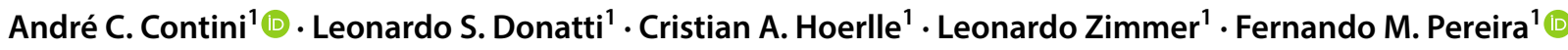

Received: 20 June 2019 / Accepted: 3 March 2020 / Published online: 24 March 2020

(C) The Brazilian Society of Mechanical Sciences and Engineering 2020

\begin{abstract}
It is still a challenging task to numerically solve flames using detailed chemical kinetics in multidimensional geometries of practical applications. To overcome this difficulty, many efforts have been done to develop chemical kinetics reducing techniques, such as ILDM, FPV, FPI, and FGM. Although these techniques are widely discussed in the literature, their implementation is not straightforward. In the present work, the FGM technique is implemented to solve a two-dimensional laminar premixed flame of $\mathrm{CH}_{4}$ /air stabilized by heat losses to the burner rim. This configuration is explored to test the FGM prediction capabilities for some stable conditions, one of them close to the blow-off limit. Temperature, mass fraction of selected species, and the burning velocity variation along the flame surface are presented, and limitations of the technique were identified by comparing FGM results against the direct integration of the full set of conservation equation. In general, the FGM technique has shown a good quantitative agreement when compared with the direct integration.
\end{abstract}

Keywords FGM $\cdot$ Heat loss $\cdot$ Laminar premixed flame $\cdot$ Burning velocity

\section{Introduction}

Many efforts have been made worldwide to reduce the fossil fuel consumption, and even so, they will remain as the main energy source for the next decades [11]. In Brazil, where the production of renewable energy is significantly higher compared with other countries, the energy from fossil fuel represented $52.5 \%$ of the energy matrix in 2016 [8]. These numbers lead to the development of new technologies and studies to enhance the combustion process efficiency.

Combustion presents a strong coupling with high nonlinear dependence among chemical, fluid dynamic, thermodynamics, and heat transfer processes. Thus, modeling flames

This article was selected from a collection of top papers presented at the 17th Brazilian Congress of Thermal Sciences and Engineering, held at Águas de Lindóia, SP, 25-28 November 2018.

Technical Editor: João Marciano Laredo dos Reis.

André C. Contini

andre.contini@ufrgs.br

1 Department of Mechanical Engineering, Federal University of Rio Grande do Sul, Rua Sarmento Leite, 425, Porto Alegre, RS 90050-170, Brazil in practical industrial applications using detailed reaction mechanisms are still prohibitive due to the high CPU time required to solve a large number of species and reactions. For this reason, there is a long-standing interest to develop reliable combustion models with high accuracy at low computation time. Chemical reduction techniques intend to meet these requirements for numerical simulations of flames. One alternative is to conduct a reduction of chemical mechanisms assuming quasi-steady-state assumption (QSSA) and partial equilibrium assumption (PEQ) $[17,18]$. Another approach is the tabulation technique, e.g., piecewise reusable implementation of solution mapping (PRISM) [24] and in situ adaptative tabulation (ISAT) [20]. These techniques store simulation results for a posteriori use. Recently, Cailler et al. [2] proposed building a chemical mechanism from scratch instead of reducing a detailed scheme. Using a novel idea, the authors rely on the use of virtual species and optimization procedure to capture the coupling between combustion and the flow field.

Other reduction methods assume that the flow timescale is much higher than the chemical time scales, and hence flow and chemistry can be decoupled. Two of the most popular applications of this assumption is the intrinsic low-dimensional manifold (ILDM) developed by [14] and the steady laminar flamelet model developed by [16]. However, the 
former is pointed out to lose accuracy in colder regions of the flame [26], whereas the latter does not have the ability to describe ignition or extinction processes.

Thereby, van Oijen and de Goey [26] proposed the flamelet-generated model (FGM) technique to overcome these problems. In the FGM framework, a database representing the combustion process is built from storing a set of laminar one-dimensional flames solved with detailed chemical kinetic (flamelets) as function of some control variables. In some cases, this methodology can be a hundred times faster than the direct integration of the conservation equation [25] without losing much accuracy. Similar approaches as the FGM are flamelet/progress variable model (FPV) proposed by [19] and the flame prolongation ILDM (FPI) proposed by [9].

The solution of premixed flame with heat loss using the FGM technique has been initially studied by [28]. The authors have explored a 2D laminar premixed flame and validated their results with a detailed model. Then, the authors explored their model in a radiating furnace with a ceramic-foam surface burner on the upper wall. The numerical simulation using FGM agreed well with experimental measurements. Donini et al. [6] presented numerical results for the DLR burner with heat loss at the walls using the FGM technique.

In the present work, the heat loss model is implemented in the FGM technique and validated by comparison with the direct integration of the full set of conservation equations. The case of study is a flame in a box similar to those studied by [28]. Two stoichiometric flames are investigated, one of them close to the blow-off limit. The results of temperature, progress variable, and mass fraction of selected species, and the prediction of the burning velocity distribution along the flame surface are presented and discussed in detail. A comparison of the predicted burning velocity along the flame surface is provided. A further verifying the agreement between the FGM and the detailed model and comparing the flame temperature along the surface of maximum heat release rate is needed. This type of detailed comparison is not found in previous studies $[4,5,9,27,28]$.

\section{Method}

\subsection{Problem definition}

In this work, premixed laminar flames of $\mathrm{CH}_{4}$ /air are modeled with the FGM technique considering heat losses. Two-dimensional planar simulations are performed. The computational domain is shown in Fig. 1 and represents a slot burner in an enclosure with an outlet at the top. The inlet is 4-mm-wide channel and the outlet $12 \mathrm{~mm}$ wide. The walls and the gas inlet are at a constant temperature

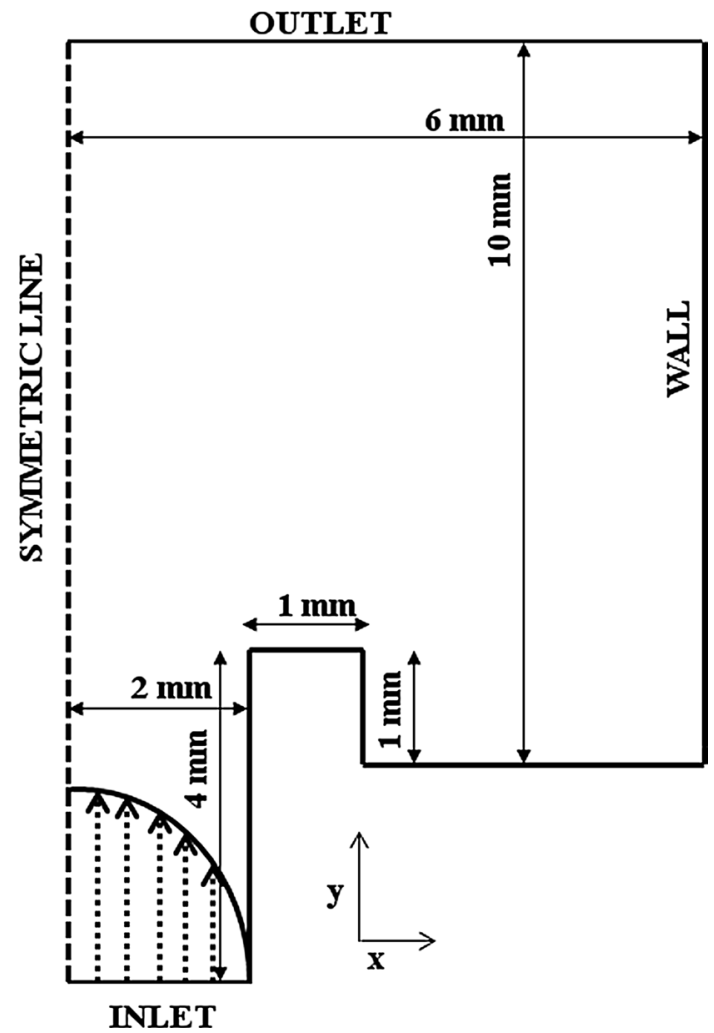

Fig. 1 The 2D planar burner configuration

(T) of $298 \mathrm{~K}$. The fresh gas velocity has a parabolic profile with a maximum value of $1.1 \mathrm{~m} / \mathrm{s}$ and $1.33 \mathrm{~m} / \mathrm{s}$ (the velocity that precedes the blow-off), equivalent to a velocity gradient at the equal to $1100 \mathrm{~s}^{-1}$ and $1330 \mathrm{~s}^{-1}$, respectively. The outlet surface is assumed to be at $1 \mathrm{~atm}$. The problem is the same test case already described by $[9,23]$.

\subsection{The FGM technique with heat losses}

The general methodology of the FGM technique is described as follows. Flamelet solutions, for a specific equivalence ratio, are obtained for different levels of enthalpy and, then, stored in a database (a manifold) as a function of some control variables. After the manifold construction is finished, multidimensional simulations are conducted solving only the conservation of total mass, momentum, and the transport equations for the control variables. Therefore, the solution of the conservation equations of each chemical species is not required as in detailed simulations. Once the convergence is achieved, the multidimensional structure of the flame is reconstructed from the manifold with the control variables solution. The flamelet equations, the appropriate definition of control variables, and the manifold generation are described in the next sections. 


\subsubsection{Manifold construction}

The initial step of the FGM methodology is to solve a set of one-dimensional flames to map the thermochemical space. The method to account for the enthalpy changes on the flames will be clearly described in Sect. 2.2.1. First, the system of conservation equations describing detailed one-dimensional laminar freely propagating premixed flames is solved through laminar flame code Chem1D [3]. The equation system accounts for conservation of total mass, chemical species mass fraction, and mixture-specific enthalpy. The conservation equationsneglecting curvature, stretch, and tangential diffusion-are solved for the steady-state regime. The boundary conditions are presented in Table 1.

Flames are computed with the DRM22 kinetic mechanism (formed by 22 species and 104 reactions) [12]. Transport properties are simplified by the unity Lewis number assumption. A diffusion velocity correction is considered for $N_{2}$ to ensure the total mass conservation. Mixture thermal conductivity and dynamic viscosity are obtained from simplified polynomial expressions as function of mixture-specific heat and temperature following [22]. Species-specific heat is obtained according to $[1,15]$. It was found that a computational domain of $2.5 \mathrm{~cm}$ discretized in 100 volumes with an adaptative mesh routine was sufficient to achieve mesh-independent solutions.

Then, any scalar $\psi$ from the flamelet solutions can be tabulated as function of the control variables. The control variables, in the present case, are the progress variable $(\mathscr{Y})$ and enthalpy $(h)$, forming a 2D manifold where $\psi=\psi(\mathscr{Y}, h)$. The progress variable describes the chemistry evolution from unburnt reactants to combustion products. According to [26], a progress variable can be represented by a linear combination of species mass fraction, $Y_{i}$.

In this work, it was sought from the literature the $\mathscr{Y}$ definition used by [5]:

$\mathscr{Y}=-\alpha_{\mathrm{O}_{2}} Y_{\mathrm{O}_{2}}+\alpha_{\mathrm{CO}_{2}} Y_{\mathrm{CO}_{2}}+\alpha_{\mathrm{H}_{2} \mathrm{O}} Y_{\mathrm{H}_{2} \mathrm{O}}+\alpha_{\mathrm{H}_{2}} Y_{\mathrm{H}_{2}}$,

where the weighting factor $\left(\alpha_{i}\right)$ is given by the inverse of the species $i$ molar mass $\left(\alpha_{i}=1 / M W_{i}\right)$. The mixture enthalpy, on its turn, maps different thermodynamic states due to heat losses to the burner walls and to the environment when radiative heat losses are taken into account. Finally, the full set of flamelet solutions are linearly interpolated into the manifold as a function of the control variables. The final manifold is discretized in 200 points in the $\mathscr{Y}$ direction and 70 points in the $h$ direction, with all points being equally

Table 1 Boundaries condition of laminar premixed flame spaced. The reader is referred to [26] for more details about the storing retrieving procedure.

Inclusion of heat losses in the manifold For predicting heat losses, the flamelets were solved for a range of enthalpies values, which implies that enthalpy becomes a control variable. The easiest way to change the mixture enthalpy is by changing the inlet boundary initial temperature. Thus, the flamelets were calculated for initial temperatures varying from $390 \mathrm{~K}$ to $240 \mathrm{~K}$ in steps of $30 \mathrm{~K}$ (following [26]). This manifold includes conditions of heat gain (initial temperature bigger than $298 \mathrm{~K}$ ) and heat loss (initial temperature smaller than $298 \mathrm{~K}$ ). However, computing flamelets for even higher heat losses by continuously decreasing the initial reactant temperature may lead to unrealistic conditions. In the literature, there is not just one method to overcome this problem [9]. In this paper, it is considered the approach of converting part of the reactants into the corresponding products but maintaining the elementary composition unaltered.

In the present work, it followed the approach of converting a fraction of the reactants $\left(\mathrm{CH}_{4}\right.$ and $\left.\mathrm{O}_{2}\right)$ into the corresponding products $\left(\mathrm{CO}_{2}\right.$ and $\left.\mathrm{H}_{2} \mathrm{O}\right)$ at the same initial temperature $(240 \mathrm{~K})$. This conversion of species respects the fuel/oxidant stoichiometric proportion whereas the mixture enthalpy (due to the low enthalpy of formation of the saturated products) and the pool of atoms of the mixture is preserved. Hence, we changed the initial reactant composition by increasing the molar fraction of $\mathrm{CO}_{2}$ and $\mathrm{H}_{2} \mathrm{O}$ in steps of 0.01 and 0.02 , respectively, (with a proportional reduction of $\mathrm{CH}_{4}$ and $\mathrm{O}_{2}$ molar fraction) until the flame extinguishes.

A scheme of the manifold including heat losses is shown in Fig. 2. Unfortunately, these two methods are not able to reproduce the cold states close to the walls, where there are combustion products at low temperatures. For this region, an extrapolation procedure is necessary [26]. Here, the lowest

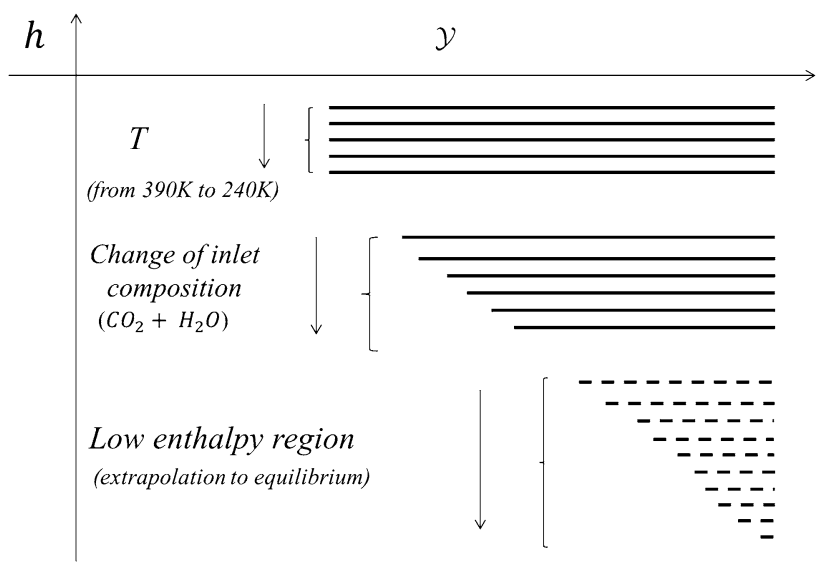

Fig. 2 Schematic representation of the 2D manifold with the different approaches for distinct regions. The range of the control variables is from -0.688 to 1.032 for the progress variable definition adopted and $-1.56 \times 10^{5}$ to $-3.00 \times 10^{6}$ for enthalpy 
enthalpy composition is approximated for the one obtained from equilibrium calculations at $298 \mathrm{~K}$ and imposing the initial composition equal to that obtained for the last flamelet solution for the maximum progress variable (Fig. 2).

\subsubsection{Multidimensional simulations}

FGM simulations in multidimensional geometries are conducted after the database (manifold) is constructed. These simulations consist in solving laminar steady-state conservation equations of total mass, momentum and a conservation equation for each control variable. Assuming unity Lewis number, the transport equations of the control variables area given by:

$\nabla \cdot(\rho \mathbf{u} \mathscr{Y})-\nabla \cdot\left(\frac{\lambda}{c_{p}} \nabla \mathscr{Y}\right)=\dot{\omega}_{\mathscr{Y}}$

$\nabla \cdot(\rho \mathbf{u} h)-\nabla \cdot\left(\frac{\lambda}{c_{p}} \nabla h\right)=0$

where $\lambda(\mathrm{W} / \mathrm{mK})$ and $c_{p}(\mathrm{~J} / \mathrm{kgK})$ are the thermal conductivity and the specific heat of the mixture, respectively. The variable $\dot{\omega}_{\mathscr{Y}}$ is the source term of the progress variable, $\mathbf{u}(\mathrm{m} / \mathrm{s})$ is the velocity vector and $\rho\left(\mathrm{kg} / \mathrm{m}^{3}\right)$ is the mixture density. The progress variable source term is defined as a linear combination of the chemical species source term that defines the progress variable.

$\dot{\omega}_{\mathscr{Y}}=\sum \alpha_{i} \dot{\omega}_{i}$

The equation system for multidimensional simulations is solved by the Fluent 16.1 software. The pressure-velocity coupling is treated by the SIMPLE method, the convective and diffusive terms are discretized by second-order schemes, and a residual error of $10^{-6}$ is assumed as convergence criterion. The FGM technique is implemented in the software via user-defined functions, including the procedure of retrieving the information from the manifold. The boundaries conditions are presented in Table 2. Mixture thermodynamic and transport properties (as viscosity conductivity and specific heat) required for the solution of the equation system are retrieved from the manifold during run-time. After the solution of the equation system, the flame structure is

Table 2 Boundary conditions imposed for $\mathscr{Y}$ and $h$ transport equations

\begin{tabular}{lll}
\hline Boundaries & $\mathscr{Y}$ & $h$ \\
\hline Inlet & $\min (\mathscr{Y})$ & $h(T=298) \mathrm{K}$ \\
Outlet & $\frac{\partial \mathscr{Y}}{\partial x_{i}}=0$ & $\frac{\partial h}{\partial x_{i}}=0$ \\
Walls & $\frac{\partial \mathscr{Y}}{\partial x_{i}}=0$ & $h(T=298) \mathrm{K}$ \\
\hline
\end{tabular}

reconstructed from the solution of control variables retrieving the temperature and the species mass fractions from the manifold. The look-up/retrieval scheme is based on bilinear interpolations between the closest values of $\mathscr{Y}$ and $h$ solution at each control volume of the computational domain.

\subsection{Determination of laminar burning velocity from the two-dimensional flames}

The two-dimensional flame analyzed in the present work is more complex than the idealized one-dimensional canonical planar adiabatic flame. It has been shown that stretch and curvature effects may significantly change the burning velocity [13]. Additionally, heat losses reduce the flame temperature, which has an exponential effect on the reaction rates and, consequently, on the burning velocity [13]. In this work, the laminar burning velocity along the flame surface is extracted in order to compare the FGM and the detailed solutions. Both results are compared with the one-dimensional laminar adiabatic flame velocity.

The laminar burning velocity $\left(S_{\mathrm{L}}\right)$ is defined at the unburnt region of the flame (cold boundary). In the twodimensional results, this region is approximated by an isothermal surface with temperature equal to $315 \mathrm{~K}$ (which represents, approximately, $1 \%$ of the adiabatic flame temperature). Points on this isothermal surface are collected by following the temperature evolution along a certain number of streamlines as shown in Fig. 3. Then, the points are used to reconstruct the surface by a polynomial fit. At this surface, the flow velocity $(\vec{v})$ is determined on its projection on the normal surface direction $(\vec{n})$ is obtained. This velocity multiplied by a temperature correction $(298 \mathrm{~K} / 315 \mathrm{~K})$ is equal to the laminar burning velocity $S_{\mathrm{L}}$.

In order to reveal the heat loss effects, a similar procedure is developed for the flame temperature. In this case, the

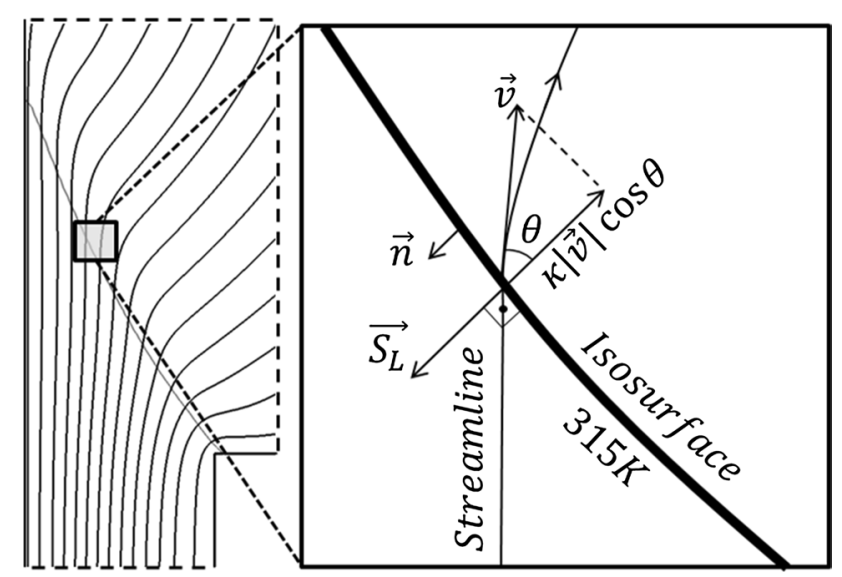

Fig. 3 Extraction of the burning velocity from the two-dimensional results ( $\kappa$ value is the ratio between $298 \mathrm{~K} / 315 \mathrm{~K}$ ) 
position of the maximum heat release rate is found for each of those streamlines and the flame temperature is collected at position. Note that this temperature is not the maximum value reached by the flame; instead, it is a temperature that characterizes the inner structure of the flame, where most reactions are happening.

\section{Results}

In order to certificate a correct implementation of FGM technique including heat losses, Sect. 3.1 presents a set of one-dimensional simulations of freely propagating $\mathrm{CH}_{4}$ /Air flames to validate the FGM technique with a detailed chemistry. In Sect. 3.2, two-dimensional results are presented. The last part, a discussion about laminar burning velocity predictions is presented to verify the capabilities of the FGM technique in predicting non-adiabatic premixed flames.

\subsection{Manifold validation}

Figure 4 shows the validation of the present implementation in the one-dimensional freely propagating flame of $\mathrm{CH}_{4} /$ air with stoichiometric equivalence ratio. The inlet temperature of the mixture is $298 \mathrm{~K}$ and atmospheric pressure is imposed. FGM results are compared with the detailed simulation (i.e., with the direct integration of the chemical kinetics and the solution of all chemical species). As explained in Sect. 2.2.1, the DRM22 kinetics mechanism is also assumed for detailed simulations. The FGM results present discrepancies in order of the $1 \%$ for both temperature and CO mass fraction when compared with the detailed simulation, suggesting that the FGM technique can adequately solve the flame and that the assumed progress variable definition is suitable to map the thermochemical space of the analyzed flame.

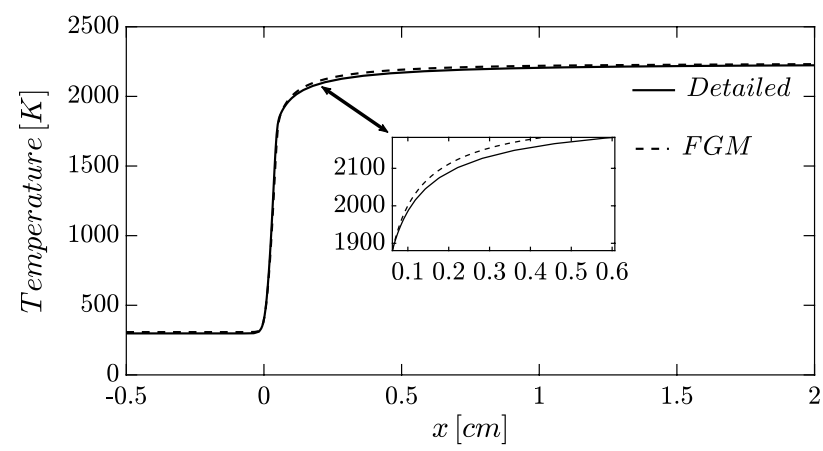

(a)

\subsection{Two-dimensional simulations}

Two-dimensional simulations were conducted for the configuration presented in Fig. 1 following the FGM methodology explained in Sect. 2.2

\subsubsection{Mesh independence test}

A mesh independence test for the two-dimensional domain is performed using the FGM technique. The computational domain is discretized with equally sized quadrilateral cells. The analysis is conducted for six different meshes from 260 to 104,000 volumes. Progress variable (Fig. 5a) and flame temperature (Fig. 5b) are chosen as parameter to evaluate the mesh independence. Maximum discrepancies are observed at $4.5 \mathrm{~mm}$ from the symmetry line. The progress variable is a scalar actually solved in the computational domain (along with enthalpy) and, consequently, have to be mesh-independent. Temperature is a scalar retrieved from the manifold.

\subsubsection{Comparison of flame structure between detailed and FGM simulations}

A comparison between detailed and FGM simulations is presented in Fig. 6 for the maximum reactants injection velocity of $1.1 \mathrm{~m} / \mathrm{s}$. In general, a good agreement between the two combustion models is found. Contours of the reaction progress variable $(\mathscr{Y})$ presents only small differences at the flame base close to the burner (Fig. 6a). At this position, the FGM simulation predicted slightly lower $\mathscr{Y}$ values. Those differences are not so evident for the contour of temperature (Fig. 6b), but they become more apparent for the $\mathrm{OH}$ mass fraction (Fig. 6c). In fact, the FGM results predicted slightly wider distributions of $\mathrm{OH}$ and $\mathrm{CO}$ (Fig. 6d) mass fractions in the thermally affected region. Despite that, scalars' maximum values and flame height are well predicted by the reduced kinetic mechanism approach.

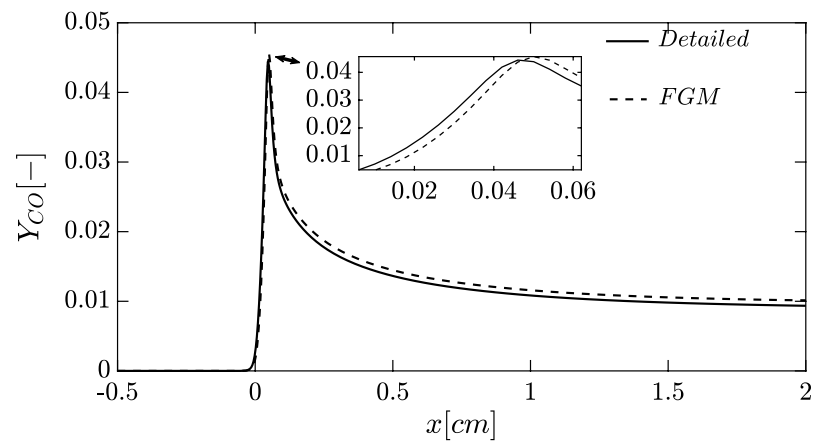

(b)

Fig. 4 Manifold validation for one-dimensional adiabatic freely propagating flame of $\mathrm{CH}_{4}$ /Air at $\phi=1.0$ and atmospheric conditions. Comparison of FGM and detailed model for $\mathbf{a}$ temperature and $\mathbf{b}$ CO mass fraction 
Major discrepancies are found for chemically slower species as can be seen in the contours of the $\mathrm{CO}$ mass fraction presented in Fig. 6d. It is observed a significant difference in the spatial distribution of the $\mathrm{CO}$ mass fractions at low temperatures close to the burner rim where the flame stabilizes. At this region, the flame extinguishes due to low temperatures next to the wall and the reactants can leak through the flame envelope without being completely burnt. The detailed simulation predicted a non-negligible concentration of $\mathrm{CO}$ around the burner, which is not predicted by the FGM simulations.

Ganter et al. [10] performed a numerical analysis of laminar methane/air side-wall-quenching. It was found that the $\mathrm{CO}$ prediction using the FGM method also presented deviations close the wall. In the same work, they evaluated the $\mathrm{CO}$ transport applying the Lagrangian analysis and showed that higher $\mathrm{CO}$ concentrations close the wall are due to diffusive transport toward that location. With the intention to capture this phenomena using the reduced technique, Efimov et al. [7] proposed a new method to evaluate the flame-wall interactions (FWI) using the FGM. Referred to as quenching flamelet-generated manifold (QFM), the focus of the study was to improve the accuracy for the prediction of $\mathrm{CO}$ near the wall in a similar geometry of the present study. They observed a strong dependence of the $\mathrm{CO}$ concentrations on the enthalpy gradients close to the wall. In order to take this dependence into account, an additional degree of freedom was included in the FGM using a second reaction progress variable. Therefore, to correctly map some species on the quenching region (near to the walls) is necessary to extend the FGM technique with additional controlling variable.

Figure 6e presents the $\mathrm{HO}_{2}$ mass fraction. Clearly, some discrepancies are noticed close to the burner rim. Popp and Baum [21] concluded that very close to the wall, in the cold region, the production $\mathrm{HO}_{2}$ occurs via the chain-breaking
Fig. 6 Comparison between detailed chemistry and FGM results. Contours of: a progress variable $\mathscr{Y}$, b temperature, c $\mathrm{OH}, \mathbf{d ~ C O}$, e $\mathrm{HO}_{2}$, f $\mathrm{HCO}, \mathbf{g ~ C H}$, and $\mathbf{h}$ normalized heat release rate

$\mathrm{H}+\mathrm{O}_{2}+\mathrm{M}=\mathrm{HO}_{2}+\mathrm{M}$. This reaction competes with the chain-branching reaction $\mathrm{H}+\mathrm{O}_{2}=\mathrm{OH}+\mathrm{O}$ for the $\mathrm{H}$ radical. However, the chain-breaking reaction described above requires no activation energy and the probability of ternary collisions is increased due to high water concentration at the wall, which has very high efficiency to promote the ternary reaction. This scenario also suggests that a quenching mechanism close to the wall acting on $\mathrm{HO}_{2}$ reactions could be captured using additional dimensions in the manifold in a similar way to the $\mathrm{CO}$ as described in [7].

The minor species $\mathrm{HCO}$ and $\mathrm{CH}_{2}$ are presented in Fig. 6f, $\mathrm{g}$, respectively. The former plays a central role in hydrocarbon combustion, whereas the latter is related PAH and $\mathrm{NO}_{x}$ formation. Theses species are well predicted by the FGM at the hot regions of the flame but, again, present important discrepancies at the cold regions with cold products showing once more the difficulty of the method in solving this condition. However, these discrepancies are non-essential to the overall flame behavior, since the FGM technique depends on the progress variable (and enthalpy) which is well-mapping the hot flame region as shown in Fig. $6 \mathrm{~h}$ for the heat release rate.

A better insight into the FGM agreement with the detailed simulation is obtained from radial profiles. Results are compared for profiles of progress variable ( $\mathscr{Y})$, temperature, and species mass fraction at $1 \mathrm{~mm}, 3 \mathrm{~mm}$, and $7 \mathrm{~mm}$ from the burner nozzle exit in Fig. 7. These positions approximately indicate the bottom, the middle of the flame, and the postflame region. Radial profiles of the progress variable and the temperature confirm the good agreement of the FGM with the detailed simulations. A negligible displacement of the

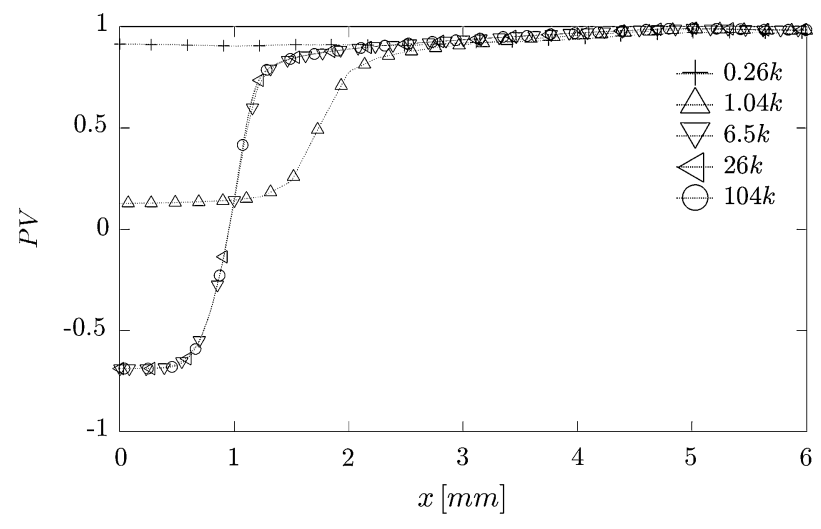

(a)

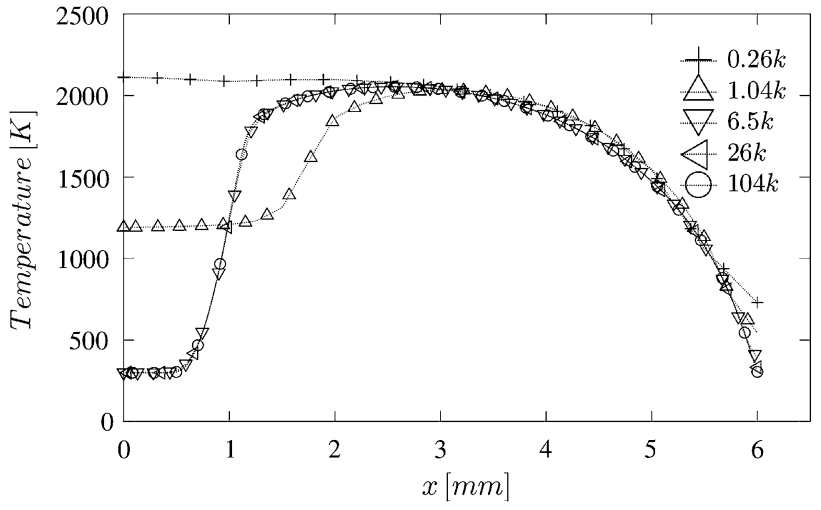

(b)

Fig. 5 Mesh independence test for the two-dimensional simulations: a progress variable profile for different meshes at $3 \mathrm{~mm}$ from the burner nozzle exit; $\mathbf{b}$ temperature profile for different meshes at $3 \mathrm{~mm}$ from the burner nozzle exit 


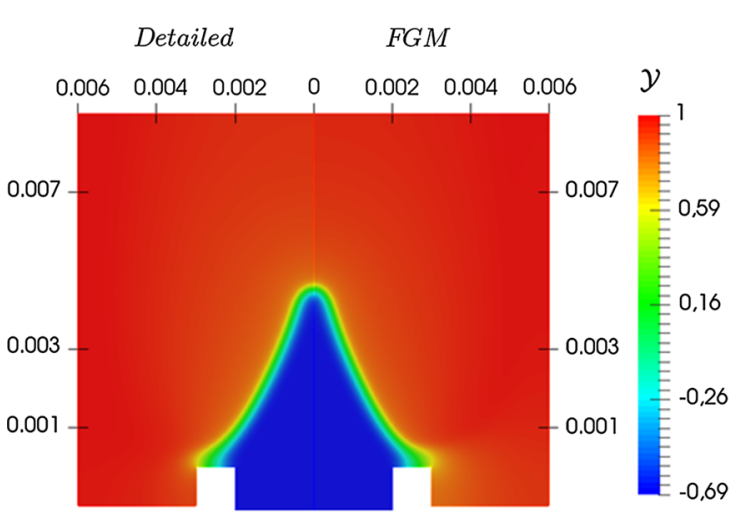

(a)

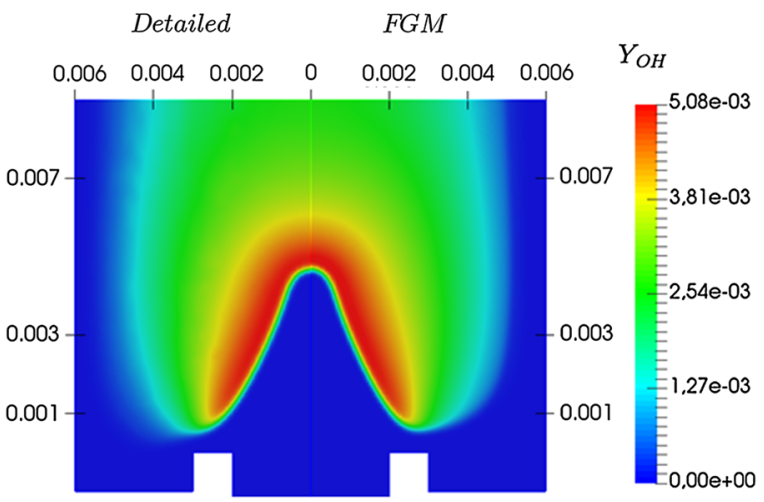

(c)

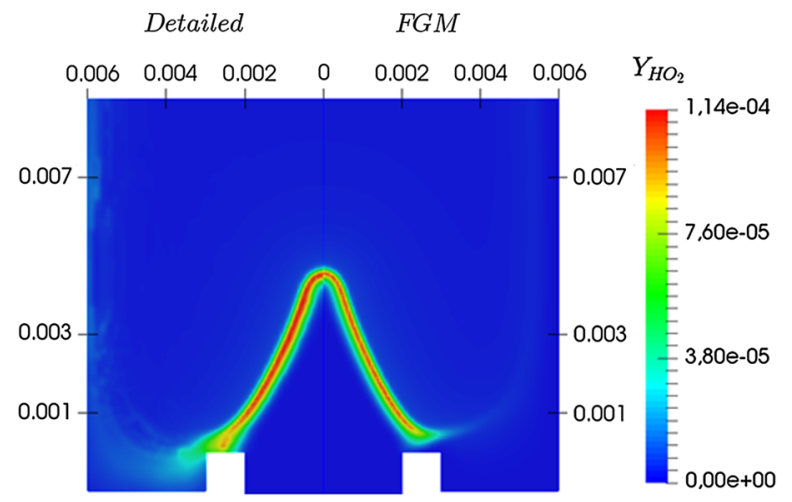

(e)

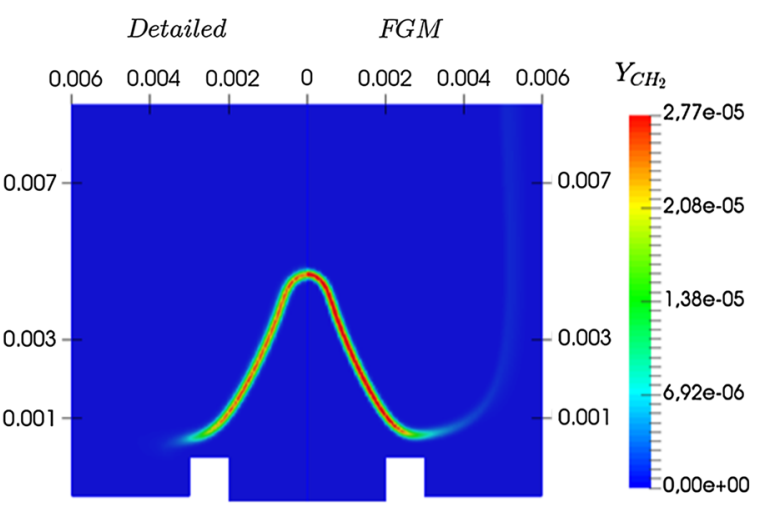

(g)

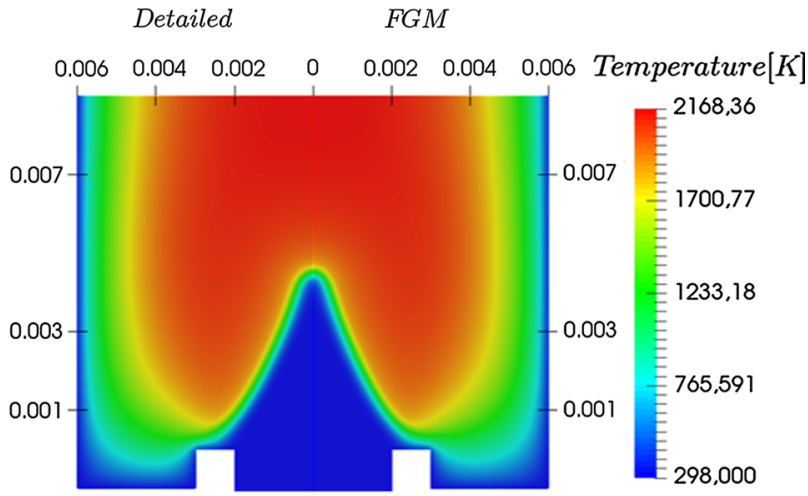

(b)

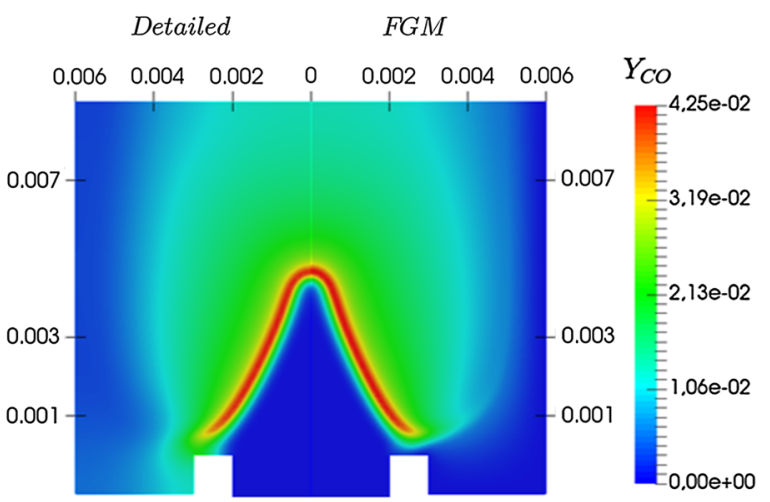

(d)

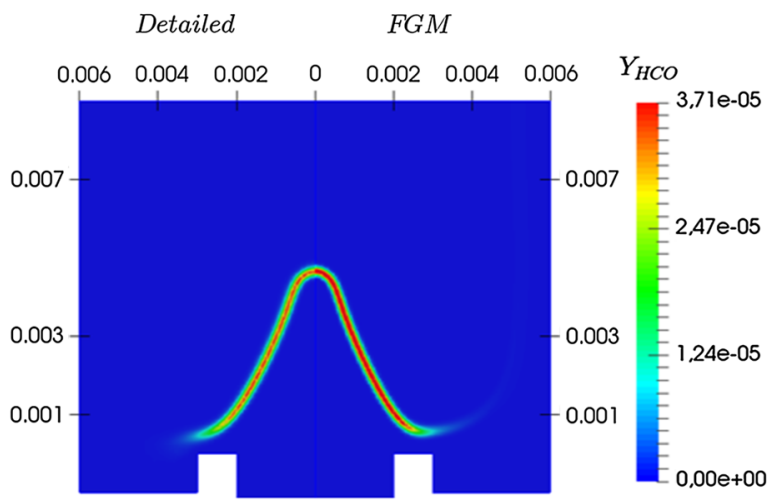

(f)

Detailed FGM

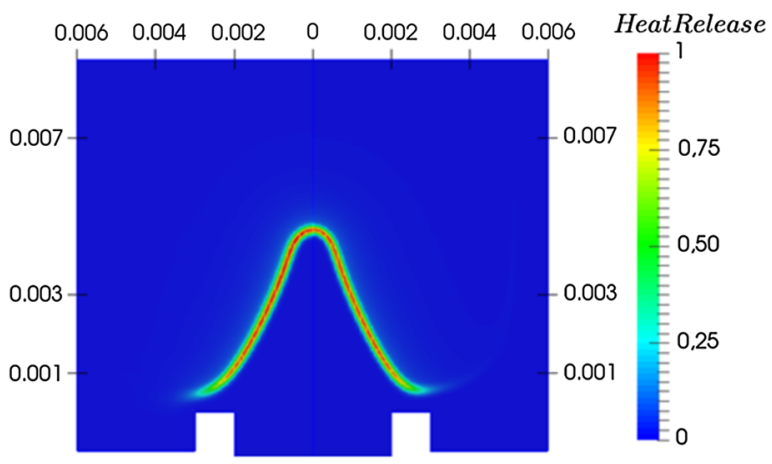

(h) 

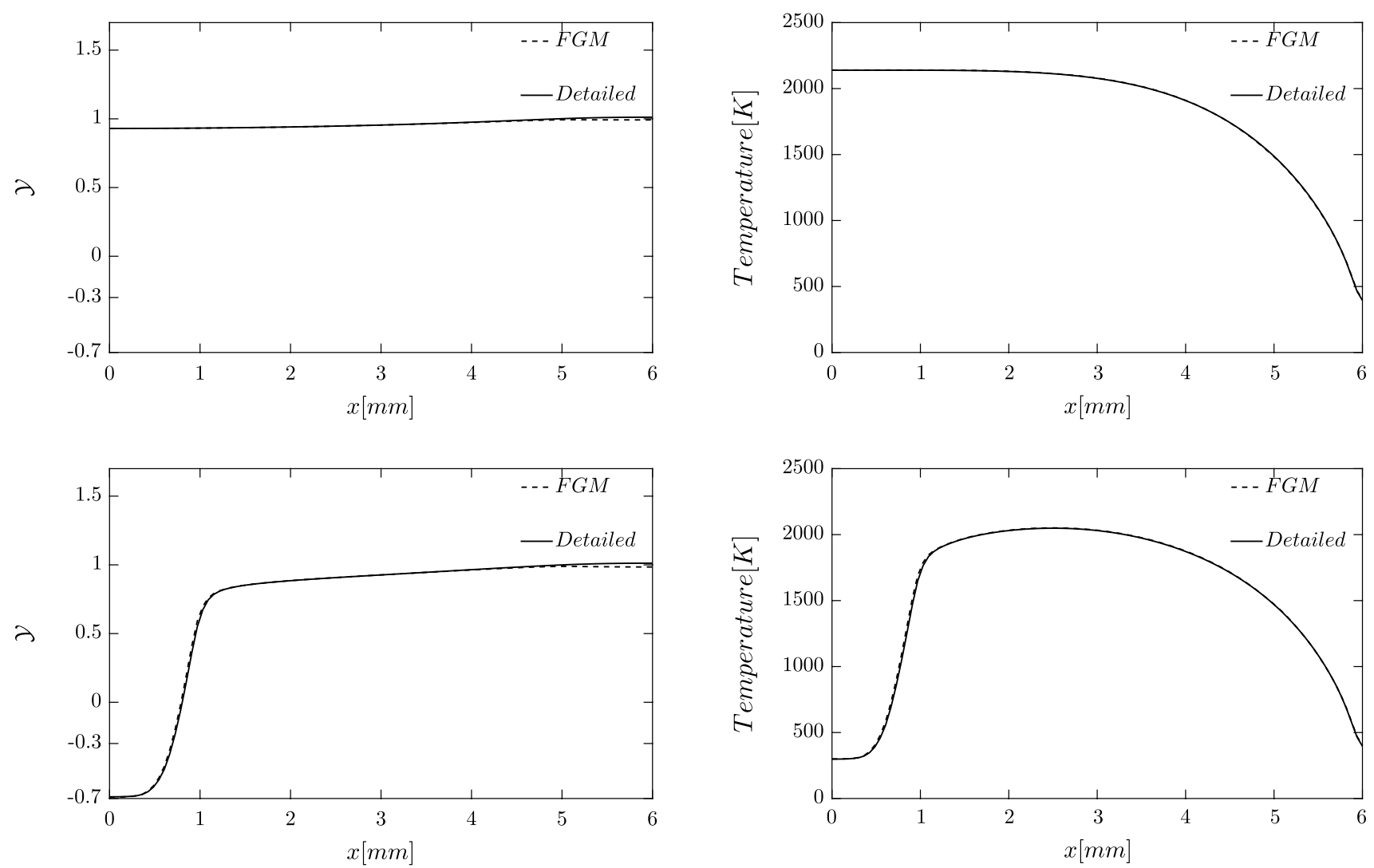

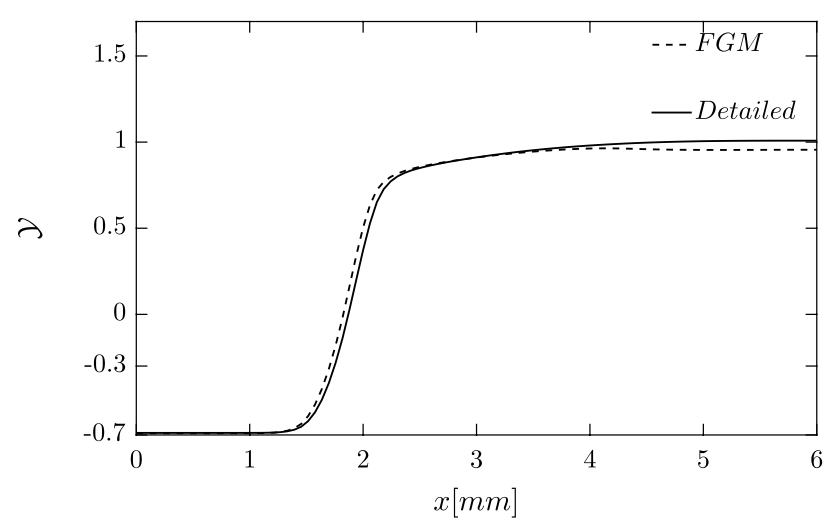

(a)

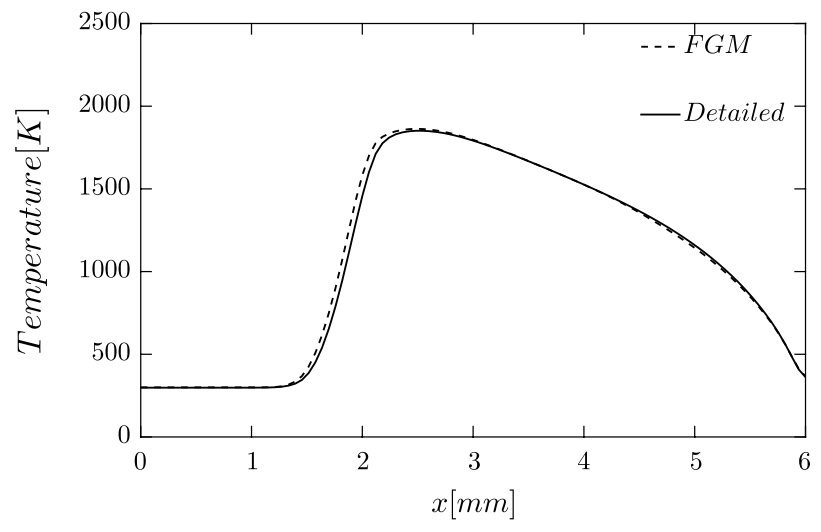

(b)

Fig. 7 Comparison of the reaction progress variable $\mathscr{Y}(\mathbf{a})$ and the temperature (b) profiles between detailed and FGM simulations at 7 mm (top), $3 \mathrm{~mm}$ (middle) and $1 \mathrm{~mm}$ (bottom) from the burner nozzle exit. Maximum reactants velocity of $1.1 \mathrm{~m} / \mathrm{s}$

FGM results is observed at the regions with higher gradients, and very small differences can be observed for the progress variable $(\mathscr{Y})$ at the maximum radial position at $1 \mathrm{~mm}$ from the burner nozzle.

A good quantitative and qualitative agreement is also observed for the major chemical species $\left(\mathrm{CO}_{2}\right)$. Figure 8a confirms the capabilities of the FGM, as simplified kinetic mechanism treatment, in predicting the major chemical species with a good accuracy. As previously reported, more important differences are observed for the $\mathrm{CO}$ mass fraction closer to the burner nozzle and the computational domain walls. Those regions are characterized by the strong heat transfer from the flame and, therefore, by the lower temperatures. Figure $8 \mathrm{~b}$ presents the minor chemical species. In general, a good agreement is found. The main difference is presented by $\mathrm{HO}_{2}$ due to the cold region close to the wall, as previously discussed.

Simulations were also performed for a slightly higher for maximum reactants injection velocity of $1.33 \mathrm{~m} / \mathrm{s}$, equivalent to a velocity gradient at wall equal to $1330 \mathrm{1} / \mathrm{s}$. 

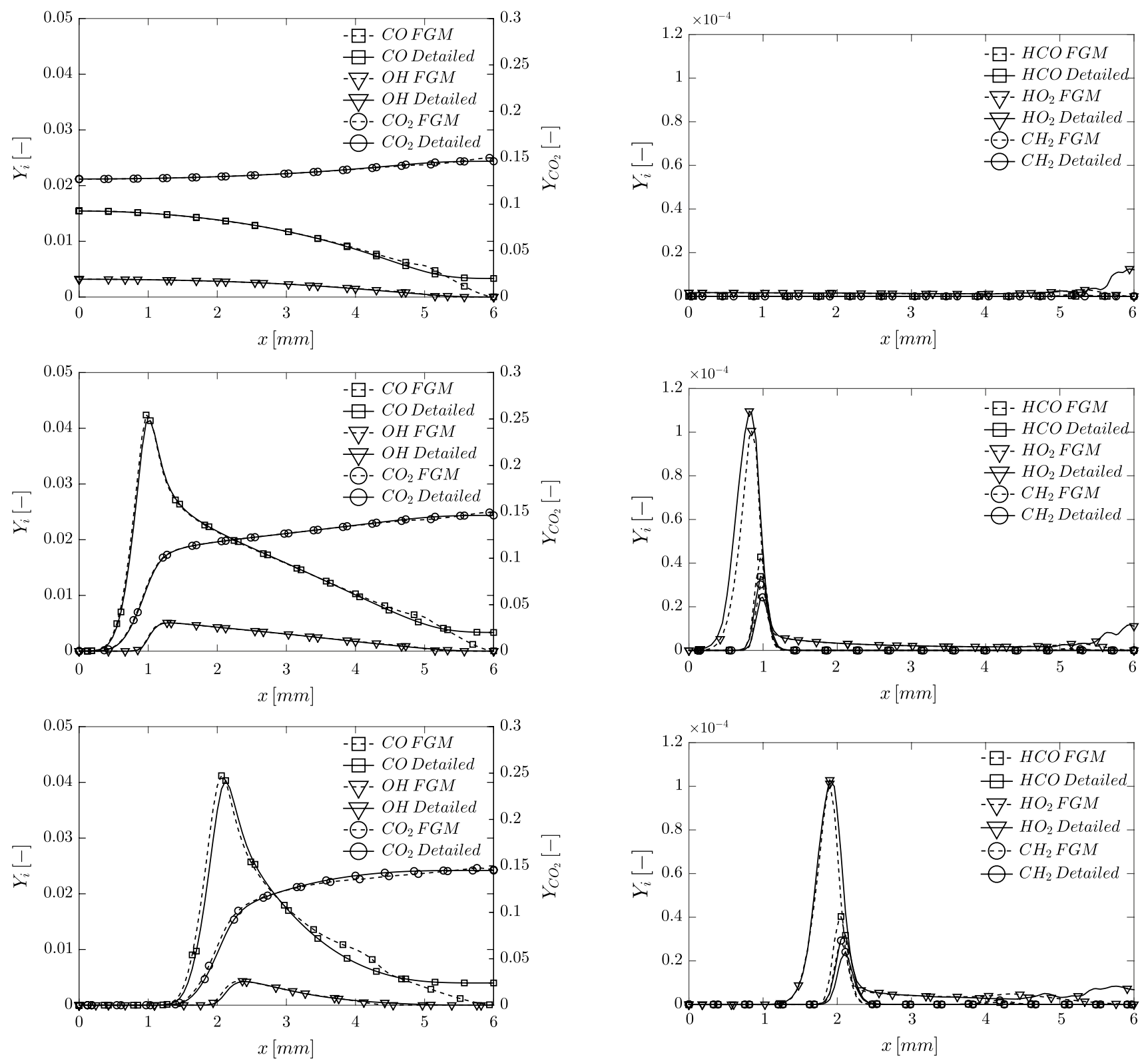

(a)

(b)

Fig. 8 Comparison of a $\mathrm{CO}, \mathrm{OH}, \mathrm{CO}_{2}$ and $\mathbf{b} \mathrm{HCO}, \mathrm{HO}_{2}, \mathrm{CH}_{2}$ mass fractions profiles between detailed and FGM simulations at 7 mm (top), $3 \mathrm{~mm}$ (middle) and $1 \mathrm{~mm}$ (bottom) from the burner nozzle exit. Maximum reactants velocity of $1.1 \mathrm{~m} / \mathrm{s}$

This maximum velocity was found to be the limit to the occurrence the flame blow-off from performing simulations increasing the maximum inlet velocity in steps of $0.01 \mathrm{~m} / \mathrm{s}$ until reach numerical instabilities. The subsequent increase of the maximum inlet velocity reduces the heat transfer from the flame to the burner until it reaches the point where the burner does not sustain the flame anymore. A comparison between detailed and FGM simulations is presented for this higher maximum inlet velocity in Fig. 9. The flame structure of this limiting case is found to be similar to the one presented in Fig. 6 but with a slightly higher flame length.
The comparison between detailed and FGM simulations also shows a similar trend as already assumed.

Despite some discrepancies for $\mathrm{CO}$ and $\mathrm{HO}_{2}$ mass fractions, the results achieved with the FGM approach presented a very good agreement with the detailed simulation but demanding only a fraction of the computational time. While detailed simulation required roughly $48 \mathrm{~h}$ to converge, the FGM methodology needed only $2.4 \mathrm{~h}$ (20 times faster) including the preprocessing step, i.e., the flamelet simulations and the manifold construction. Also, it should be noted that the manifold needs to be created just once for 


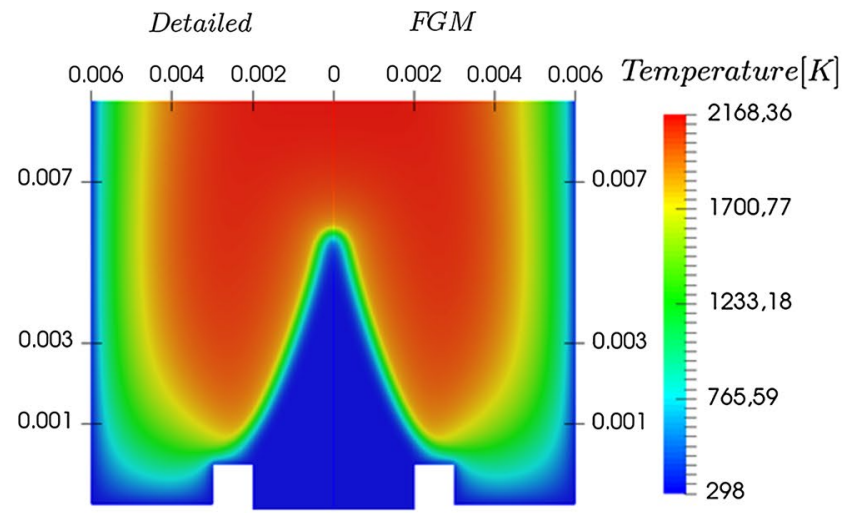

(a)

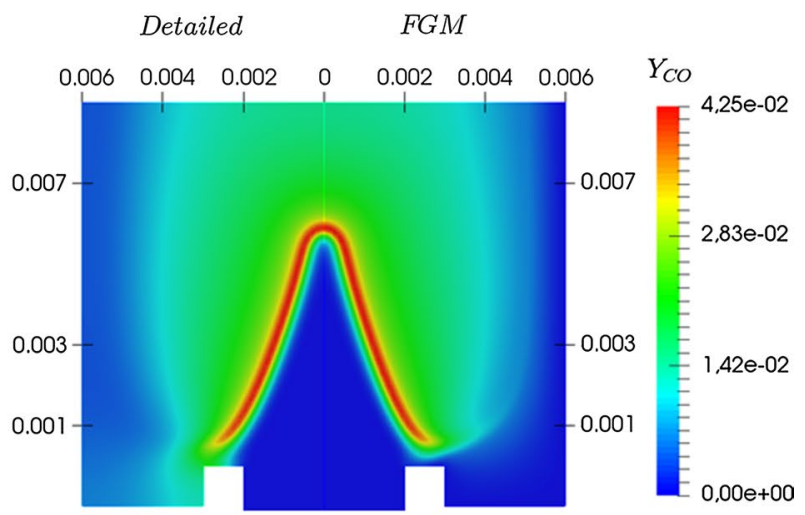

(b)

Fig. 9 Contours of temperature (a) and (b) CO mass fraction for detailed and FGM simulations for the maximum reactants inlet velocity of $1.33 \mathrm{~m} / \mathrm{s}$

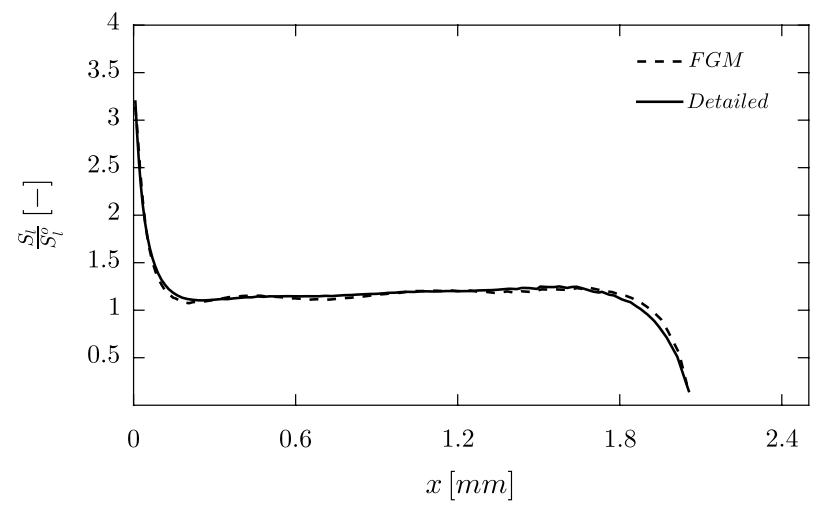

(a)

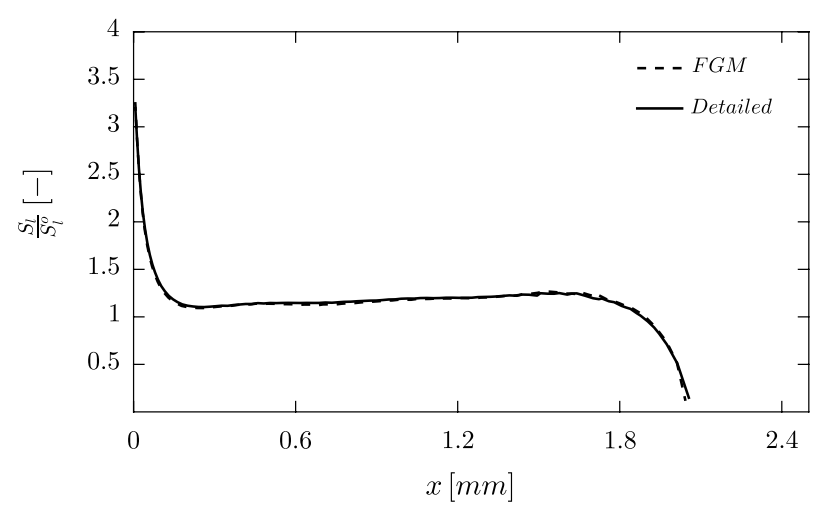

(c)

Fig. 10 Burning velocity (a, c) along the flame surface normalized by the $1 \mathrm{D}$ burning velocity $\left(S_{l}^{o}=29.72 \mathrm{~cm} / \mathrm{s}\right)$ and temperature $(\mathbf{b}, \mathbf{d})$ at the maximum heat release rate position normalized by the same

each equivalence ratio under consideration. In other words, the same manifold can be used to perform simulations of different burners subjected to different heat loss conditions.

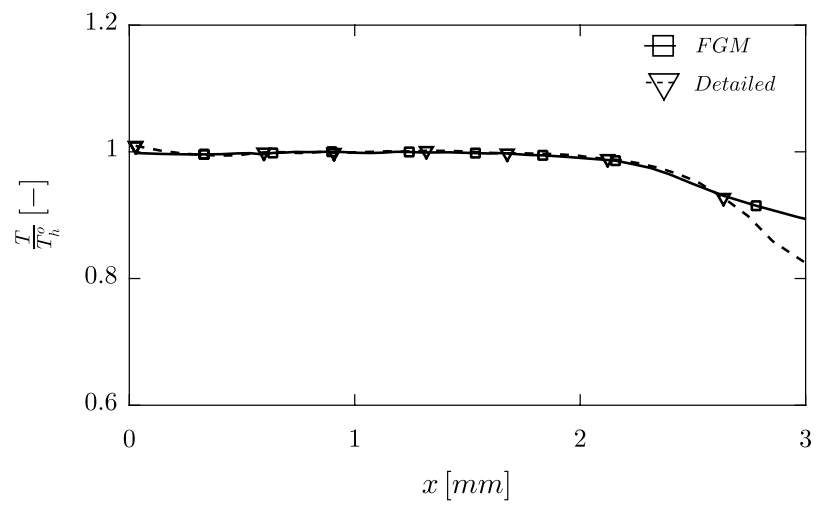

(b)

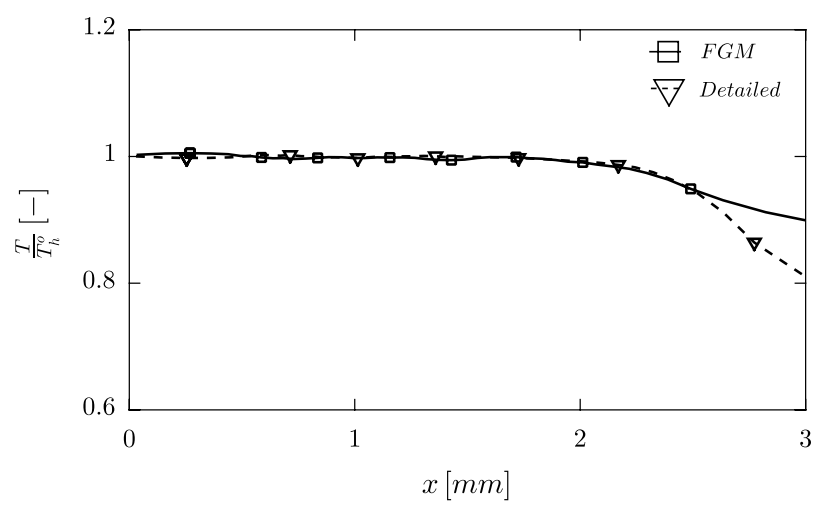

(d)

temperature from a $1 \mathrm{D}$ adiabatic simulation $\left(T_{h}^{o}=1721 \mathrm{~K}\right)$. Reactants inlet velocity of $1.10 \mathrm{~m} / \mathrm{s}(\mathbf{a}, \mathbf{b})$ and of $1.33 \mathrm{~m} / \mathrm{s}(\mathbf{c}, \mathbf{d})$

\subsection{Evaluation of the laminar flame speed}

Figure 10a shows the burning velocity along the flame surface (the coordinate $x$ is the horizontal axis) for the maximum inlet velocity of $1.1 \mathrm{~m} / \mathrm{s}$. Three distinct regions are 
observed: one close to the burner rim, which is affected by heat losses, an intermediary region, which approaches the 1D results, and a third region close to the centerline, where the flame is affected by curvature effects. The FGM and the detailed results are very similar showing, again, the good approximation obtained with the reduced technique. The behavior at the flame tip can be explained by the analysis of the [13] for the burning velocity at low stretch rates which show that

$\frac{S_{l}}{S_{l}^{o}}=1-\delta_{u}^{o} \nabla \cdot n$

were $\delta_{u}^{o}$ is the flame thickness and $\nabla \cdot n$ represents the flame curvature (which is equal to $-2 / R_{\mathrm{f}}$, were $R_{\mathrm{f}}$ is the flame surface radius). Thus, the burning velocity is increased at the tip of the conical flame due to pure curvature effects. Note that stretch effects are not present since in our simulations, and it was imposed unitary Lewis number.

Figure $10 \mathrm{~b}$ presents the flame temperature at the maximum heat release rate position. It can be seen that as the distance increases, the temperature at the reaction region is reduced due to heat losses to the burner rim. This explains the decrease in the burning velocity in this region. Far from the burner walls, the temperature of the reaction region reaches a plateau close to its adiabatic value. This is why the burning velocity approaches $S_{l}^{o}$ at the intermediary region and increases due to pure curvature effects at the flame tip.

In Fig. 10b, it is possible to see some important differences between both numerical methods. The temperature decrease near the cold wall is more intense for the detailed simulation because the flame edge stabilizes closer to the burner rim when compared to the FGM model. From Fig. $6 \mathrm{~h}$, it is possible to verify that the flame envelope for the detailed formulation is anchored closer to the burner rim. It can be occurring due to recurring difficulty of the FGM in capture regions where large gradients of enthalpy are present, as discussed by [7].

Figure 10c, $d$ shows the same results for the maximum inlet velocity of $1.33 \mathrm{~m} / \mathrm{s}$. The FGM is still capable of reproducing the detailed results with very good accuracy. The flame behavior at this limiting stabilization point presents the same characteristics already discussed.

\section{Conclusions}

In this paper, an implementation of the FGM technique for premixed flames with heat losses is compared to the detailed solution of the conservation equations. The problem is a premixed laminar flame stabilized on a slot burner. The FGM technique presented very good qualitative and quantitative results when compared to the reference model even for a flame close to the blow-off limit. The main discrepancies occurred due to difficulties in mapping the thermochemical state of the system near the walls where combustion products at low temperature are found.

A detailed comparison of the burning velocity along the flame surface revealed the effects of the heat loss to the burner rim and the pure curvature effect at the flame tip. The present study explores the Le $=1$ condition. For fuels with Lewis numbers far from the unity, another formulation of the FGM would be necessary to take preferential diffusion into account. In the present case, the FGM technique was able to capture the flame behavior with good agreement with the detailed model.

Acknowledgements The authors acknowledge the support of Coordenação de Aperfeiçoamento de Pessoal de Nível Superior - Brazil (CAPES).

\section{Compliance with ethical standards}

Conflict of interest The authors declare that they have no conflict of interest.

\section{References}

1. Burcat A, Gardiner WC (2000) Ideal gas thermochemical data for combustion and air pollution use. In: Gas-phase combustion chemistry. Springer, pp 489-538

2. Cailler M, Darabiha N, Veynante D, Fiorina B (2017) Building-up virtual optimized mechanism for flame modeling. Proc Combust Inst 36(1):1251-1258

3. Chem1D A (n.d.) One-dimensional Laminar flame code. Eindhoven University of Technology

4. de Goey L, Boonkkamp JTT (1999) A flamelet description of premixed laminar flames and the relation with flame stretch. Combust Flame 119(3):253-271. https://doi.org/10.1016/S0010 -2180(99)00052-8

5. Donini A, Bastiaans R, van Oijen J, de Goey L (2015) Differential diffusion effects inclusion with flamelet generated manifold for the modeling of stratified premixed cooled flames. Proc Combust Inst 35(1):831-837. https://doi.org/10.1016/j.proci.2014.06.050

6. Donini A, Bastiaans RJ, van Oijen JA, de Goey LPH (2015) The implementation of five-dimensional FGM combustion model for the simulation of a gas turbine model combustor. ASME Paper No. GT2015-42037

7. Efimov DV, de Goey P, van Oijen JA (2019) OFM: quenching flamelet-generated manifold for modelling of flame-wall interactions. Combust Theory Model 24(1):72-104

8. EPE (2017) Brazilian Energy Balance 2016. Empresa de Pesquisa Energética, Rio de Janeiro

9. Fiorina B, Baron R, Gicquel O, Thevenin D, Carpentier S, Darabiha $\mathrm{N}$ et al (2003) Modelling non-adiabatic partially premixed flames using flame-prolongation of ILDM. Combust Theory Model 7(3):449-470

10. Ganter S, Straßacker C, Kuenne G, Meier T, Heinrich A, Maas U, Janicka J (2018) Laminar near-wall combustion: analysis of tabulated chemistry simulations by means of detailed kinetics. Int J Heat Fluid Flow 70:259-270

11. IEA (2017) Key world energy statistics. International Energy Agency, Paris 
12. Kazakov A (1995) Drm22, vol 21. University of California at Berkeley, Berkeley, p 2017

13. Law C (2006) Combustion physics, 1st edn. Cambridge University Press, Cambridge

14. Maas U, Pope SB (1992) Simplifying chemical kinetics: intrinsic low-dimensional manifolds in composition space. Combust flame 88(3):239-264

15. McBride BJ, Gordon S, Reno MA (1993) Coefficients for calculating thermodynamic and transport properties of individual species. National Aeronautics and Space Administration, Office of Management, vol 4513

16. Peters N (1984) Laminar diffusion flamelet models in nonpremixed turbulent combustion. Prog Energy Combust Sci 10(3):319-339. https://doi.org/10.1016/0360-1285(84)90114-X

17. Peters N (1985) Numerical and asymptotic analysis of systematically reduced reaction schemes for hydrocarbon flames. In: Roland G, Bernard L, Roger T (eds) Numerical simulation of combustion phenomena. Springer, Berlin, Heidelberg, pp 90-109

18. Peters N, Rogg B (1993) Reduced kinetic mechanisms for applications in combustion systems. Lecture notes in physics, vol 15. Springer

19. Pierce CD, Moin P (2004) Progress-variable approach for largeeddy simulation of non-premixed turbulent combustion. J Fluid Mech 504:73-97

20. Pope SB (1997) Computationally efficient implementation of combustion chemistry using in situ adaptive tabulation. Combust Theor Model 1(1):41-63

21. Popp P, Baum M (1997) Analysis of wall heat fluxes, reaction mechanisms, and unburnt hydrocarbons during the head-on quenching of a laminar methane flame. Combust Flame 108(3):327-348

22. Smooke MD, Giovangigli V (1991) Premixed and nonpremixed test problem results. In: Reduced kinetic mechanisms and asymptotic approximations for methane-air flames. Lecture note in physics. Springer, Berlin, pp 29-47

23. Somers LM, De Goey L (1995) A numerical study of a premixed flame on a slit burner. Combust Sci Technol 108(1-3):121-132

24. Tonse SR, Moriarty NW, Brown NJ, Frenklach M (1999) Prism: piecewise reusable implementation of solution mapping. An economical strategy for chemical kinetics. Isr J Chem 39(1):97-106

25. van Oijen JA (2002) Flamelet-generated manifolds: development and application to premixed laminar flames. Ph.D. thesis, Eindhoven University of Technology

26. van Oijen JA, de Goey LPH (2000) Modelling of premixed laminar flames using flamelet-generated manifolds. Combust Sci Technol 161(1):113-137

27. van Oijen J, de Goey L (2002) Modelling of premixed counterflow flames using the flamelet-generated manifold method. Combust Theory Model 6(3):463-478

28. van Oijen J, Lammers F, de Goey L (2001) Modeling of complex premixed burner systems by using flamelet-generated manifolds. Combust Flame 127:2124-2134

Publisher's Note Springer Nature remains neutral with regard to jurisdictional claims in published maps and institutional affiliations. 